\title{
Axisymmetric Tandem Mirrors: Stabilization and Confinement Studies
}

R.F. Post, T.K. Fowler, R. Bulmer, J. Byers, D. Hua, and L. Tung

This article was submitted to Open Magnetic Systems for Plasma Confinement, Novosibirsk, Russia, July 5-9, 2004

\section{July 14, 2004}




\section{DISCLAIMER}

This document was prepared as an account of work sponsored by an agency of the United States Government. Neither the United States Government nor the University of California nor any of their employees, makes any warranty, express or implied, or assumes any legal liability or responsibility for the accuracy, completeness, or usefulness of any information, apparatus, product, or process disclosed, or represents that its use would not infringe privately owned rights. Reference herein to any specific commercial product, process, or service by trade name, trademark, manufacturer, or otherwise, does not necessarily constitute or imply its endorsement, recommendation, or favoring by the United States Government or the University of California. The views and opinions of authors expressed herein do not necessarily state or reflect those of the United States Government or the University of California, and shall not be used for advertising or product endorsement purposes.

This is a preprint of a paper intended for publication in a journal or proceedings. Since changes may be made before publication, this preprint is made available with the understanding that it will not be cited or reproduced without the permission of the author.

This report has been reproduced directly from the best available copy.

Available electronically at http://www.doc.gov/bridge

Available for a processing fee to U.S. Department of Energy

And its contractors in paper from

U.S. Department of Energy

Office of Scientific and Technical Information

P.O. Box 62

Oak Ridge, TN 37831-0062

Telephone: (865) 576-8401

Facsimile: (865) 576-5728

E-mail: reports@adonis.osti.gov

Available for the sale to the public from

U.S. Department of Commerce

National Technical Information Service

5285 Port Royal Road

Springfield, VA 22161

Telephone: (800) 553-6847

Facsimile: (703) 605-6900

E-mail: orders@ntis.fedworld.gov

Online ordering: http:/ / www.ntis.gov/ordering.htm

\section{OR}

Lawrence Livermore National Laboratory

Technical Information Department's Digital Library

http:/ / www.llnl.gov/tid/Library.html 


\title{
AXISYMMETRIC TANDEM MIRRORS: STABILIZATION AND CONFINEMENT STUDIES
}

\author{
R. F. Post, T. K. Fowler, R. Bulmer, J. Byers, D. Hua, and L. Tung
}

Lawrence Livermore National Laboratory: 7000 East Ave, L-644, Livermore, CA 94551,post3@llnl.gov

The "Kinetic Stabilizer" has been proposed as a means of MHD stabilizing an axisymmetric tandem mirror system. The $K-S$ concept is based on theoretical studies by Ryutov, confirmed experimentally in the Gas Dynamic Trap experiment in Novosibirsk. In the K-S beams of ions are directed into the end of an "expander" region outside the outer mirror of a tandem mirror. These ions, slowed, stagnated, and reflected as they move up the magnetic gradient, produce a low-density stabilizing plasma.

At the Lawrence Livermore National Laboratory we have been conducting theoretical and computational studies of the K-S Tandem Mirror. These studies have employed a low-beta code written especially to analyze the beam injection/stabilization process, and a new code SYMTRAN (by Hua and Fowler) that solves the coupled radial and axial particle and energy transport in a $K-S T$ M. Also, a "legacy" MHD stability code, FLORA, has been upgraded and employed to benchmark the injection/stabilization code and to extend its results to high beta values.

The FLORA code studies so far have confirmed the effectiveness of the $K-S$ in stabilizing high-beta (40\%) plasmas with stabilizer plasmas the peak pressures of which are several orders of magnitude smaller than those of the confined plasma. Also the SYMTRAN code has shown D-T plasma ignition from alpha particle energy deposition in $T-M$ regimes with strong end plugging.

Our studies have confirmed the viability of the K-S T$M$ concept with respect to $M H D$ stability and radial and axial confinement. We are continuing these studies in order to optimize the parameters and to examine means for the stabilization of possible residual instability modes, such as drift modes and "trapped-particle" modes. These modes may in principle be controlled by tailoring the stabilizer plasma distribution and/or the radial potential distribution.

In the paper the results to date of our studies are summarized and projected to scope out possible fusionpower versions of the $K-S T-M$

\section{INTRODUCTION}

Dating back to the 1960 s, fusion research has provided examples of axisymmetric "open" confinement devices in which radial transport rates approached the classical "Spitzer" level, i.e., situations in which turbulence, if present at all, was at too low a level to adversely affect the radial transport. If such lowturbulence conditions could be achieved in a Tandem Mirror system it could lead to fusion power systems that would be much simpler and require much less time to develop than those based on closed-field confinement, e.g., the tokamak, where the transport is known to be dominated by turbulence. Since axisymmetric mirror systems are prone to MHD interchange instabilities the key to exploiting this new opportunity is to find a practical way to stabilize such modes. The Kinetic Stabilizer [1] represents one possible avenue to achieving this goal.

The starting point for the K-S concept is a theoretical analysis by Ryutov [2]. He showed that plasma contained in an axisymmetric mirror cell can be MHD-stabilized by the presence of effluent plasma on the expanding field lines outside the mirrors: This stabilization was conclusively demonstrated in the Gas Dynamic Trap (GDT) experiment at the Budker Institute in Novosibirsk, Russia, up to mirror-cell plasma beta values of $40 \%$ [3].

The GDT operates in a high-collisionality regime, one in which the plasma leaking through its mirrors, though much lower in density than the confined plasma, is sufficiently high to stabilize the confined plasma. This situation would not be the case in a high-confinement $\mathrm{T}$ $\mathrm{M}$ system. The K-S resolves this dilemma by employing ion beams injected up the magnetic gradient in the "expander" region outside the outermost mirror in such a way that as they are compressed, stagnated, and reflected they form a "stabilizer" plasma in the expander.

The concept of the T-M, based on the idea of suppressing the losses through the mirrors of a fusion confinement region by creating regions of increased positive electrical potential in "plug" cells at the ends, has evolved over time since its origination in the mid-1970s by Dimov [4] in Russia and Fowler and Logan [5] in the U.S. That first version of the T-M involved the straightforward idea of generating the required elevated potential in the plugs simply by maintaining an order-ofmagnitude higher density in the plugs than that of the fusion plasma in the central cell. However, since that time the T-M concept has changed radically from its original form. 
To review briefly the history of the development of the T-M up to the present time, it has been constrained to the use of non-axisymmetric fields in order to insure MHD stability. This constraint has, in turn, led to a progressive increase in complexity of the T-M concept, involving introducing such entities as "thermal barriers" (localized regions of negative-going potential acting as barriers to electron flow between the central cell and the plugs). The origin of the need for more complex versions lies in the circumstance that when non-axisymmetric fields are employed in the plugs of a T-M the peak magnetic fields are limited (limiting the mirror ratios) so that in attempting to implement the first, simple version of the T-M the plasma volume in the plugs cannot be made small enough to satisfy power balance and engineering requirements. From this dilemma there arose the need for thermal barriers. In addition to the economic and engineering penalties, the use of non-axisymmtric fields in a T-M leads to enhanced cross-field particle transport arising from bounce-resonant drifts of the mirror-reflected ions.

Should it turn out to be feasible to stabilize T-M systems with axisymmetric magnetic fields it would then be possible to design K-S T-M fusion systems based on the original T-M concept. When employing axisymmetric fields the plug mirror fields may be made much larger (up to 25 Tesla with present technology), while at the same time the volume of the plug cells can be made much smaller than is possible with non-axisymmetric fields. As a result the penalty of limited plug mirror fields and large plug plasma volume no longer applies, relaxing the engineering constraints and eliminating the need for thermal barriers.

\section{ORIGINS OF THE KINETIC STABILIZER CONCEPT}

The Kinetic Stabilizer idea evolved from an earlier idea, the Kinetic Tandem [6]. The Kinetic Tandem concept was proposed as a means to utilize the tandem mirror idea in a confining field having the form of a long solenoid whose axisymmetric field is constant in the confinement region, and then decreases uniformly at each end (i.e., no mirrors are employed). The confining field is itself MHD stable, every field line having positive curvature. To create the potential peaks needed to confine the fusion plasma, ion beams are injected up the magnetic gradient at the ends. The ion beams are aimed at small angles to the field lines so the ion density is increased greatly (relative to that at the ion sources) by magnetic compression and by the ions being slowed and reflected as they approach the top of the magnetic gradient. These localized density peaks thus generate a positive ambipolar confining potential by the same Boltzmann-like mechanism as that employed in the original tandem mirror concept. Analytical and computer-code calculations were performed that demonstrated the generation of fusion-relevant plasma parameters using realistic ion beam current densities and beam energies. Although the Kinetic Tandem appeared to be capable of becoming a fusion power system, it came at a price: in order to "pay" for the beam power required to maintain the plugs the length of the central solenoid had to be many kilometers, casting doubt on the economic feasibility of the idea.

The Kinetic Stabilizer borrows the ion-beamproduced plasma peak idea of the Kinetic Tandem and marries it to the MHD stabilization technique demonstrated in the GDT experiment. That is, in a mirror or tandem mirror system constructed using only circular coils, ion beams are aimed up the magnetic gradient at the ends so as to form a plasma density peak in the region of positive field-line curvature outside the outermost mirror. This peaked plasma density then stabilizes the confined plasma by its presence. As will be shown, the beam power requirements of the Kinetic Stabilizer are orders of magnitude lower than the beam powers needed for the Kinetic Tandem. The most significant point is, however, the earlier-mentioned one that the Kinetic Stabilizer offers a way to design mirror or tandem-mirror systems using only axisymmetric fields.

\section{OVERVIEW OF KINETIC-STABILIZER STUDIES AT LLNL}

For the past two-and-one-half years theoretical and computational studies have been underway at the Laboratory directed at assessing the viability of the K-S concept as applied to fusion-relevant axisymmetric T-M systems. Three different computer codes have been employed in carrying out these studies, including two that were written specifically to model specific plasma physics aspects of the K-S T-M.

The first one of these special codes was written using the Mathematica ${ }^{\circledR}$ platform. The purpose of this code was to provide a flexible means for calculating the flux surfaces of the mirror and "expander" magnetic fields and then to use these calculated fields and flux surfaces to evaluate the MHD-stability integral contributions (in the low-beta limit) from both the K-S plasma and from the plasma confined in the plugs and central cell plasmas. This code in its various specialized forms proved to be a very useful tool for scoping the parameter space of different expander and plug and central cell field configurations.

The second code that was employed, one that was benchmarked at low beta against the just-described code, was the MHD-stability code FLORA, written in the 1980s [7]. This code, which includes finite-beta and finite-orbit effects provides a versatile platform for the analysis of the K-S T-M. 
The third code that has been employed, SYMTRAN, is a coupled radial/axial transport code written specially for the analysis of axisymmetric T-M systems. It couples the effects of radial transport ("classical" plus ETGenhanced at the present) with that associated with radially dependent axial confinement from the plug potentials, calculated using the Pastukhov/Cohen formulation [8].

Earlier publications $[1,9,10]$ have given the results of first studies of the K-S T-M. In the sections to follow we will describe new results that have been obtained, including those from the new SYMTRAN transport code, recent results from the FLORA MHD code, and calculations prompted by the need to address the suppression of residual instability modes, such as "trapped-particle" instabilities.

\section{THE SYMTRAN CODE}

The SYMTRAN code is an adaptation of an earlier code, SPHERE, for spheromaks, now modified for tandem mirror physics. Motivated by the K-S concept, it is an extension of the earlier TAMRAC rate-equation code [11] that omitted radial transport but which successfully accounted for experimental results in TMX $[12,13]$. The SYMTRAN code differs from the earlier tandem mirror radial transport code TMT [14] in that it is focused on axisymmetric tandem mirrors and classical diffusion, whereas TMT emphasized non-ambipolar cross-field transport in the experiments TMX and MFTFB arising from the use of "yin-yang" coils in the plugs and non-symmetric transitions between the plugs and an axisymmetric center cell. Both codes exhibit interesting but different non-linear behavior.

While pulsed operating scenarios may be of interest, the calculations with SYMTRAN have first focused on achieving a steady-state fusion power system. They have also focused on classical diffusion, which is possibly achievable in tandem mirrors as noted below. Examples of ignited steady states with classical radial diffusion were found. The highest fusion power gain achieved so far, not yet optimized, is $Q \approx 10$.

Since it turns out that the highly non-linear tandem mirror model used does not always yield a steady state, a study has been undertaken to determine conditions for a steady state. First, one notes that convergence problems in an earlier steady-state code appear to be solved in the time-dependent version. Secondly, there is sometimes encountered a thermal instability common to all fusion reactors. Criteria for thermal stability are developed that explain the apparent stability of TMX and predict stable regimes for reactors. Finally, and most importantly, it is found that achieving steady state can require auxiliary heating that impacts the fusion power gain $Q$.

The requirement for auxiliary heating appears to arise from the properties of classical transport near a cold boundary, usually at the plasma edge analogous to the "scrapeoff" in a tokamak. However, unlike a tokamak in which the scrapeoff occurs at a well-defined location outside the magnetic separatrix, in a tandem mirror scrapeoff-like zones can also occur internally, yielding multiple hot cylinders separated by thin cold zones where temperatures plunge and end plugging is lost. With further work, it may be shown to be possible to provide the stabilizing power only by the end plugs, with externally controlled feedback to prevent instability.

The design requirements to avoid plasma microinstabilities in the end plugs and in the center cell have been considered, complimentary to the other studies reported here of the use of the K-S in the MHD stabilization of the end plugs. Maintaining plug stability sets requirements on the magnetic field and radius in the plug. For the center cell, the most prominent microinstability possible is one known from tokamaks -the ion temperature gradient (ITG) mode. This mode is expected to be stabilized by the tandem mirror potential, as noted below. The code includes a model of electron thermal gradient (ETG) transport thought to persist in tokamaks when not subject to the ITG mode.

Figure 1 shows results of confinement in a tandem mirror reactor center cell, using the SYMTRAN code with 1-D radial transport and formulas to represent end losses with self-consistently calculated potentials. Radial transport of the ions is assumed to be classical, based on the success in tokamaks whereby ITG modes are stabilized by electric field shear. Electric field stabilization can more easily be achieved in a tandem mirror, utilizing the natural potential and voltages applied to segmented end plates to shape the profile as needed to stabilize both ITG and rotational modes. As noted the code does include "anomalous" electron heat transport (in gyro-Bohm form) to represent ETG modes that are one candidate for residual electron anomalous transport in tokamaks. Even so, an electron temperature of $80 \mathrm{KeV}$ and a fusion power gain $Q=10$ have been obtained, not yet optimized. Here $B=3 \mathrm{~T}$ and $\mathrm{R}$ (solenoid radius) $=1$ $\mathrm{m}$, giving $B R=3$ Tesla-meters (comparable to that for the poloidal field in ITER but much less than the $B R=15$. value for the toroidal field). Note that these results were obtained using the original tandem mirror concept as it was demonstrated in TMX, one that does not require "thermal barriers" to achieve end plugging.

The code predictions of a high electron temperature reached in the ignited state deserve special comment. The heating that produced these temperatures K-S T-M comes from two sources. The first of these is the ion-electron energy transfer from the high-energy ions in the plugs. The reason for its importance: the K-S T-M has no thermal barrier, the plasma density in the plugs is an order of magnitude higher than the central cell plasma density, and its ions have an order of magnitude higher energy than those of the central cell. The second source of electron heating is, of course, energy transfer from the 
alpha particles of the D-T reaction, the energy that leads to ignition and that perpetuates the burn.

These heating sources would be ineffective in maintaining a high electron temperature if excessive electron heat transport along the field lines and through the outer mirror were to occur. However, in the K-S T-M configuration this energy transfer is inhibited for two reasons. The first of these is the ambipolar potential of the plasma, a natural consequence of the quasineutrality constraint, which operates directly to inhibit electron heat losses.

The second of these processes has to do with inhibiting the cooling effect that would result from the return of secondary electrons produced by particle bombardment of the ends of the chambers. This inhibition comes from the large field expansion in the expander region. The effect represents a further bonus from employing an axisymmetric mirror geometry in which the emerging field lines expand out to a radius that

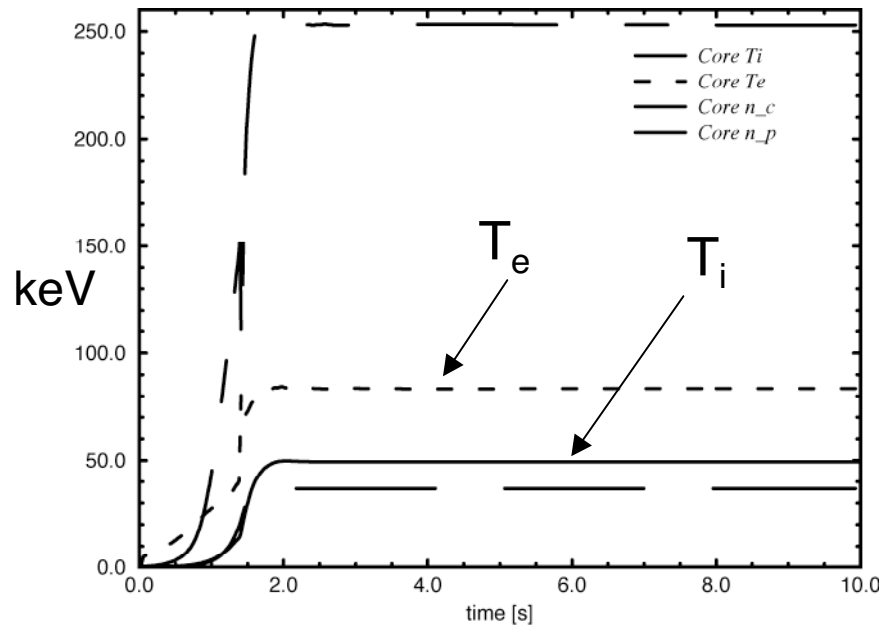

Time (sec.) is very large compared to the central cell plasma radius. As was shown theoretically by Ryutov and Mirnov [15], under these circumstances the electron population becomes completely decoupled from the end walls as regards thermal conduction effects, allowing the electron temperature to rise to high values without excessive power losses. In their theory the critical expansion ratio is given by the square-root of the ratio of the ion mass to the electron mass. This important new item of mirror plasma physics, including confirmation of the value of the critical expansion ratio, was validated in the GDT experiment. Since the plasma conditions in the expander of the K-S T-M are likely to differ from those in the GDT, it remains to be shown that the same mechanism will operate there. However, the concept involved, i. e., creating a potential distribution at a position of large expansion ratio that turns back secondary electrons, should be an attainable objective.

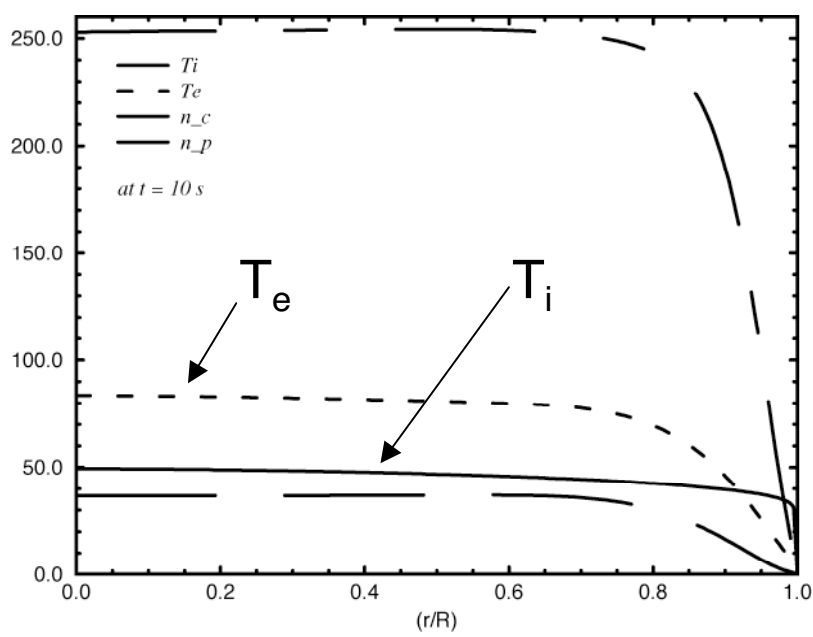

$r / R$

Fig. 1. (Left) Computer-generated plot of $T_{e}$ and $T_{i} \underline{v s}$ time, showing ignition and stable burn of a D-T plasma. (Right) Plot of the same parameters as a function of the plasma radius.

\section{EXPANDER DESIGN ISSUES AND K-S PLASMA REQUIREMENTS}

In considering the design of the expander for a K-S $\mathrm{T}-\mathrm{M}$ there are several issues that must be considered. The most important one is, of course, that of maximizing the positive contribution to the MHD stability integral. However, this maximization must be consistent with other constraints, such as avoidance of the "firehose" mode that could be stimulated in the expander by the presence of the $\mathrm{K}-\mathrm{S}$ plasma. Another is the less-well-defined one of creating K-S plasma regimes that provide sufficient "communication" to the plasma that is to be MHDstabilized (i.e. the plug and central-cell plasmas) to avoid the appearance of "trapped-particle" modes in those plasmas. These constraints will require compromises between conflicting requirements and will require innovative solutions.

As an aid in understanding the issues involved we repeat here the MHD stability integral in the form given by Ryutov [2] in his original discussion of the stabilization effect of plasma in the expander region of a mirror-confined plasma.

$$
I_{S}=\int_{-L}^{L} a^{3} \frac{d^{2} a}{d z^{2}}\left[p_{\text {perp }}+p_{\text {par }}+\rho v^{2}\right] d z>0, \text { Stable }
$$


Here $a(\mathrm{~m})$ is the plasma radius and $L(\mathrm{~m})$ and $-L(\mathrm{~m})$ represent the extreme ends of the plasma region, i.e. the outer boundaries of the expander regions. The negative (destabilizing) contributions to the integral come only from those regions of the plug and mirror cells where the field lines are concave toward the axis. Ryutov and Mirnov [15] have shown how to shape the vacuum-field flux surfaces in these regions in an optimum way, i.e. how to minimize the contributions from the regions of negative curvature. In a similar way it is possible to maximize the positive contribution to the integral of the expander and the K-S plasma through shaping the field and tailoring the axial distribution of the K-S plasma through control of injection angles and other means. This latter optimization must, however, take into account the other constraints mentioned above.

As an example of this optimization process, carried out with consideration of other constraints, we consider the "double-conical" expander flux surface, illustrated schematically in Figure 2.

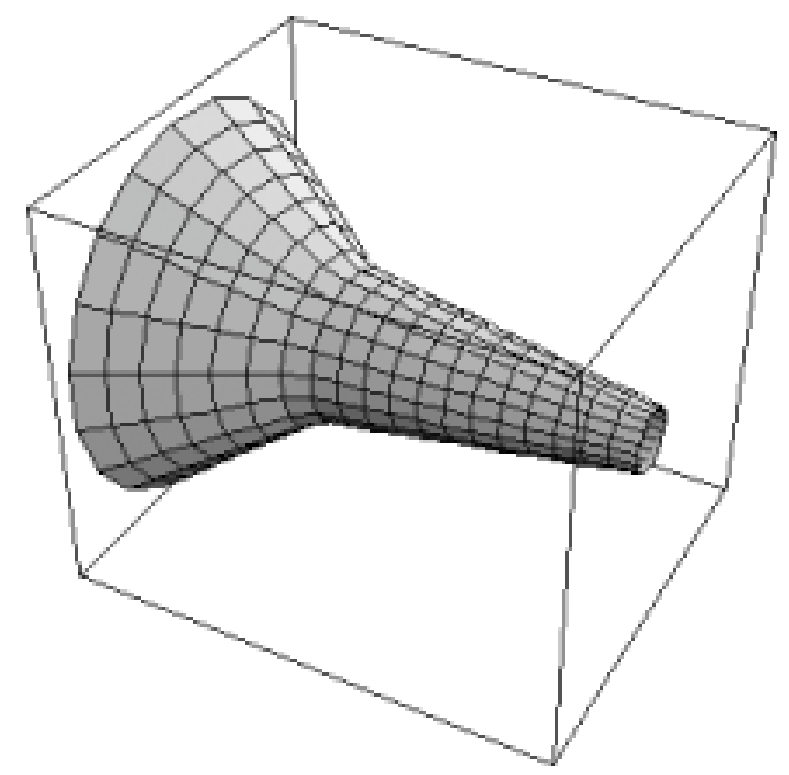

Figure 2. Schematic plot of double-conical expander consisting of two conical flux surfaces with a transition region of large positive curvature between them

One of the constraints on the double-conical expander is to insure that the beta values of the K-S plasma are small compared to the beta values in the plug and central cell. In this way one can avoid excessive field-line distortions that would otherwise arise in operation. Ryutov [16] has derived an approximate scaling law for this situation that insures that this constraint on the relative beta values will be satisfied. The condition is given in Equation 2 below.

$$
\frac{\beta_{\mathrm{K}-\mathrm{S}}}{\beta_{\text {plug }}}=2 \alpha \frac{\mathrm{a}_{\mathrm{K}-\mathrm{S}}}{\mathrm{L}_{\text {plug }}}, \text { Stable }
$$

Here $a_{\mathrm{K}-\mathrm{S}}(\mathrm{m})$ is the mean radius of the transition region in the expander, $L_{\mathrm{plug}}(\mathrm{m})$ is the length between the plug mirrors, and $\alpha$ is a constant of order unity.

Not only is the control of the shape of the flux surface in the expander a means for optimizing the performance of the K-S, but also the means by which the $\mathrm{K}-\mathrm{S}$ plasma is generated plays a critical role. As an example, ongoing theoretical studies are examining the conditions required to insure suppression of "trappedparticle" modes in the plug and central plasma. Such modes can arise when there is inadequate "communication" along the field lines between the plasmas that are to be MHD-stabilized by the K-S plasma and the K-S plasma itself. In the Gas Dynamic Trap it appears from the robust MHD stabilization that is observed that the effluent plasma, though much lower in density than the mirror-confined plasma, is still able to provide sufficient communication between the interior regions and the expander region to avoid trapped-particle modes. In the K-S a different situation prevails in that the process of generating the stabilizing plasma involves external means, means that do not necessarily provide a communicating plasma link between the confined plasmas and the K-S plasma.

While our theoretical studies to date have not as yet provided firm criteria for the plasma bridge between the confined plasma and an externally generated K-S plasma, there are nevertheless some qualitative considerations that can be taken into account to move in the right direction. One of these concerns the way in which the ion beams are injected into the expander. In previous reports $[1,9,10]$ the injection angles of the beams have been chosen to be off-axis with respect to the local direction of the field lines, with angles chosen so that all of the injected ions are reflected before the peak of the mirror is reached. While this has advantages for certain expander flux surface contours it is not an essential feature of the injection. In matter of fact the instability integral code, when programmed to accept injected ion angular distributions centered on the local field line direction at the point of injection ("parallel" injection), shows very good results, with an added bonus. In these cases the "wings" of the angular distribution represent ions that are reflected early on in moving up the magnetic gradient. Because of the $a^{3}$ scaling of the stability integral, Equation 1, these ions make a large positive contribution to the integral. At the same time, those ions that are aimed more nearly along the field lines penetrate deeply into the expander, approaching the mirror. At the same time their density increases markedly (relative to that at the point of injection) owing to their being slowed down in forward motion and being compressed magnetically by 
the converging field. These ions (and their accompanying electrons) thus can help in the formation of a plasma bridge between the stabilizing plasma far out on the expander and the mirror-confined plasma.

As an example of the order of the effects involved the stability integral code was run for a case where the plug magnetic field was generated by two solenoidal coils with a 1.0 meter inner radius and a length of 1.0 meter, with their centers separated by a distance of 2.5 meters. In this example the "expander" field was simply the fringing field from this two coils. Figure 3 shows a 3-D plot of the flux surface of this field configuration and Figure 4 shows the calculated on-axis magnetic field of the two coils. The angular distribution that was assumed is shown in Figure 5. Assuming unit ion pressure at the surface from which the ions are injected, located at a distance of 20 meters from the plug cell, Figure 6 is a plot of the ion pressure as a function of axial position in the expander. In this calculation the effect of a rising plasma potential as one approaches the mirror has not been taken into account as this effect is dependent on such factors as the injected ion energy and the details of the density variation near the mirror of the confined plasma. However, as a rough estimate will reveal the corrections for potential effects will be significant only at the innermost points of the plot.

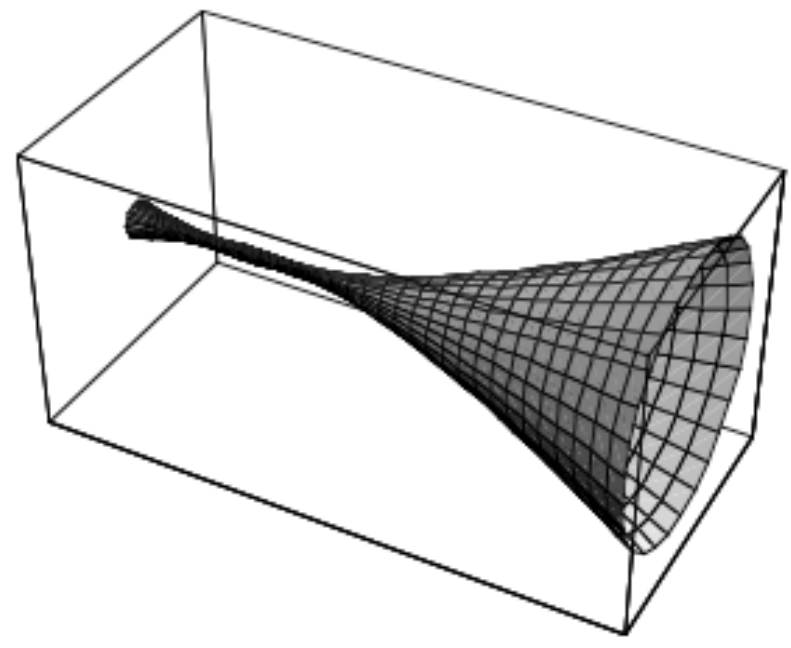

Figure 3. Computed flux surface of a high-field plug mirror cell and its fringing field

The upshot of the results just described is that the "transition" region between the plug plasma and the K-S plasma will be shortened markedly by "parallel" injection as compared to off-angle injection of the K-S ions. This circumstance should make it simpler to establish an adequate plasma bridge between the two regions, should it prove to be necessary. Among the ways this might be accomplished, as was suggested in a previous report [10], is to use gas jets injected at the inner edge of the transition region, ionized by the plug plasma. Other means might include the injection of a small component of ions with a narrower angular distribution and higher energies than the main K-S ions, with angles and energies chosen so that the tip of this group penetrates all the way to the inner boundary of the plug plasma.

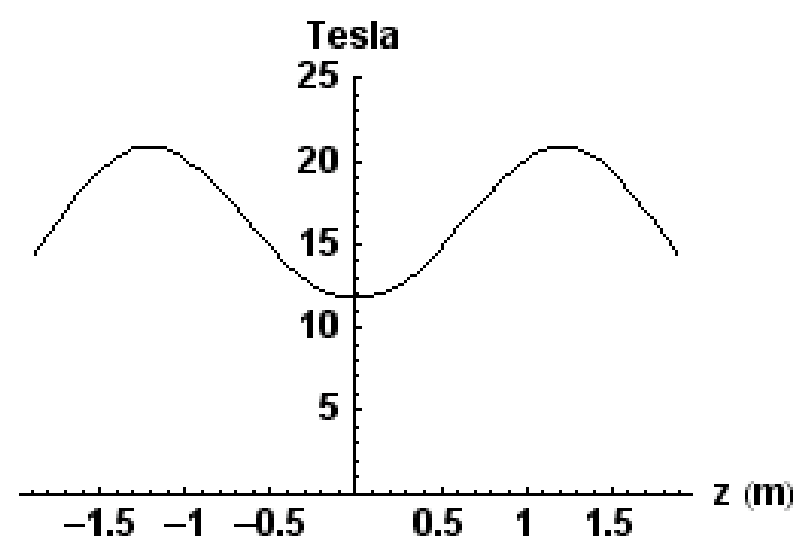

Figure 4. Calculated on-axis magnetic field of a high-field plug cell

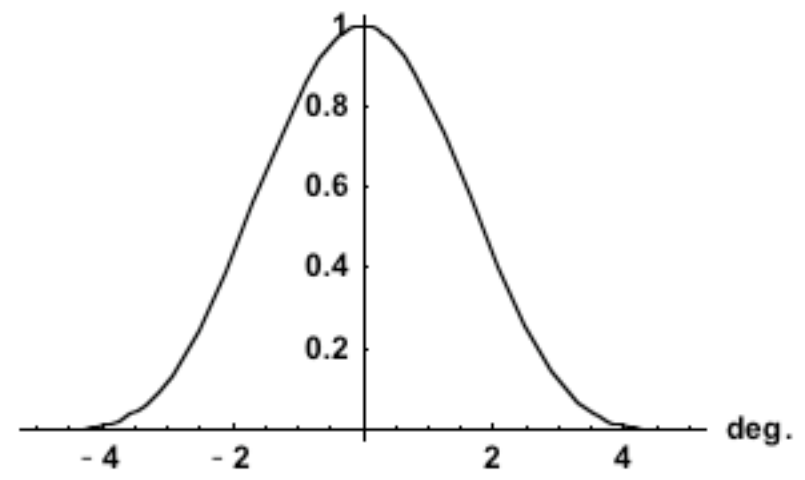

Figure 5. Angular distribution of ion-injected K-S ions

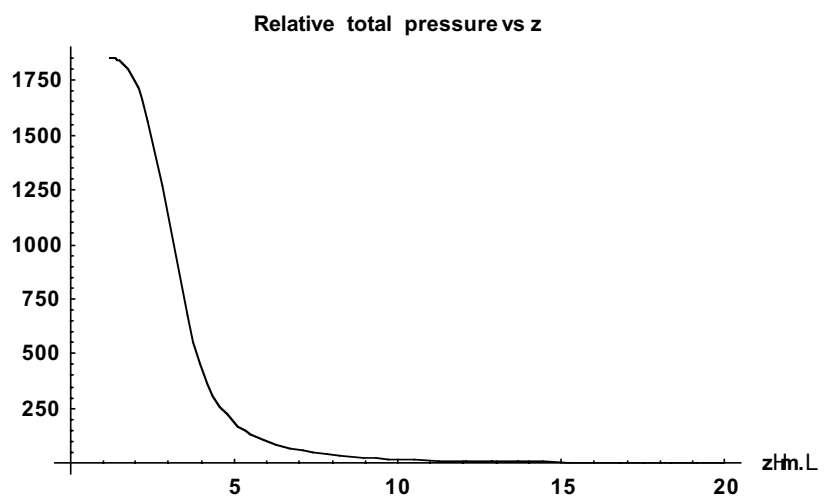

Figure 6. Total K-S plasma ion pressure as a function of position

To conclude the discussion of the above example of "parallel" injection, the plot in Figure 7 shows the value of the integrand of the stability integral as a function of 
position, assuming unit ion pressure at the surface on which the ion sources are located. Note the large contribution to the integral at large $\mathrm{z}$ values, alluded to above. It should also be noted that the ratio of the absolute value of the stability integral (Equation 1) when evaluated for the K-S plasma and for the plug cell, when evaluated for a unit peak-pressure "normal mode" distribution, has the value $4.1 \times 10^{5}$. This result implies that the ion pressure averaged over the K-S injection surface can be nearly six orders of magnitude smaller than that in the plug cell and still perform its MHD stabilization role. With optimization of the expander flux surface even higher ratios would be achieved.

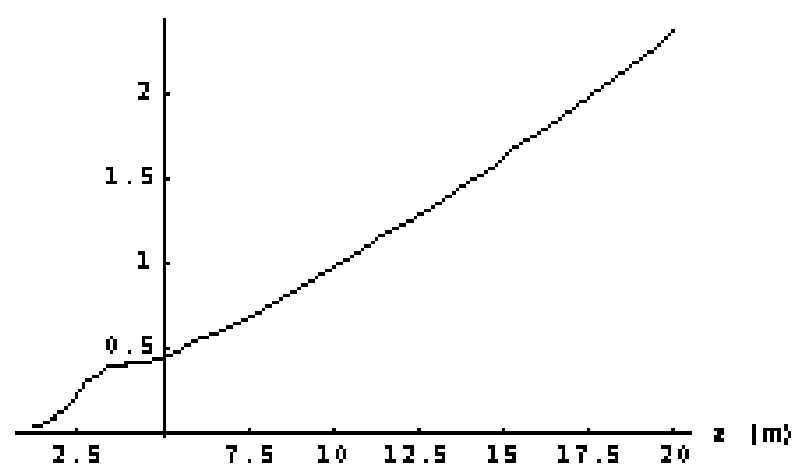

Figure 7. Calculated integrand of K-S stability integral

\section{OTHER POSSIBLE AVENUES TO THE USE OF THE KINETIC STABILIZER MECHANISM}

The discussion to this point has been mainly concerned with the use of injected ion beams to implement Ryutov's stabilization mechanism in tandem mirror systems. However, as shown by its first demonstration in the Gas Dynamic Trap experiment, there are other ways to accomplish the desired result. We have noted that a "high-confinement" plasma regime, such as that in a fusion tandem mirror device, differs fundamentally from the "high-collisionality" regime of the GDT in the density of the effluent plasma in the expander, whence the idea of using ion beams to create a stabilizer plasma. However, Ryutov's stabilization mechanism is a very general one and there are other ways that it might be implemented in a tandem-mirror context that should not be overlooked.

In previous papers two "other ways" that were mentioned to create or to enhance the stabilizer action included ECRH heating of the electrons of the K-S plasma, since the positive contribution of the K-S plasma to the stability integral is proportional to the total kinetic pressure of that plasma - ions plus electrons. Already alluded to in Section $\mathrm{V}$ in another context is the possibility of creating a stabilizing plasma stream in the expander by the use of gas jets or low-energy neutral beams injected on the outer slope of the plug potential hill. After the plasma has been built up stably by the use of K-S beams, the gas jets could take over and the beams could be turned off.

There is yet another possibility, one that it appears could be implemented in a full-scale tandem-mirror fusion power system. While the SYMTRAN code calculations were aimed at plasma ignition and operation at high fusion- $Q$ values, this is not the only possible operating regime for tandem mirror systems. It has long been recognized that mirror-based fusion-power systems could be operated in a low- $Q$, "driven" mode [17] if they employ direct converters at their ends to recycle the energy carried out by the unburned fuel ions (and the plug ions of a TM). In this operating regime a new possibility arises: if special attention is paid to the minimization of the negative contributions to the stability integral of the plug and central cell, an "auto-stabilization" analogous to that occurring naturally in the GDT can occur. This possibility is a direct consequence of the fact that an axisymmetric tandem-mirror fusion system can employ very high magnetic fields in the plugs, so that the radius of the plasma in the plugs is much smaller than that of the plasma in the central cell. Thus, even though the plasma pressure that must be stabilized in the plugs is much higher than that in the central cell, the nozzle-constricted pressure of the end-loss ions escaping from the central cell, over the plug potential barriers and through the mirrors, can be high enough to MHD-stabilize both the plugs and the central cell in cases of practical interest.

Minimization of the negative contribution of the plugs to the stability integral can come not only from employing high fields in the plug but also from shaping the flux surfaces and operating with "sloshing ions" in the plugs [9]. Maximization of the positive contributions to the integral from the presence of ions escaping out the ends can be accomplished also by shaping the flux surfaces in the expander.

To illustrate the numbers involved in a fusionrelevant example, the following values for the two segments of the stability integral (plug and expander were calculated: for the plug, with a length between the mirrors of 3.0 meters, a plasma radius at the mirrors of $0.2 \mathrm{~m}$., and with a sloshing-ion distribution having unity peak pressure, $I_{\text {plug }}=-0.000034$. For the expander, with unit pressure of escaping ions at the peak of the outermost mirror, $I_{\text {expander }}=+0.43$, so that the ratio of their absolute value is $1.3 \times 10^{4}$. In this example the ion pressure of the escaping ions at the mirror throat can thus be more than four orders of magnitude smaller than that of the plug plasma and still stabilize it.

If we use previously cited high- $Q$ results from SYMTRAN (Section III) to estimate the magnitude of these "auto-stabilization" effects we find using the above stability integral ratio that the exiting ions from the 
central cell fail by about a factor of five to stabilize the plug plasma (which makes the largest negative contribution to the stability integral). However, even without attempting to further optimize the expander or plug cell parameters in order to improve the stability integral ratio, this level of auto-stabilization should be enough to stabilize a low- $Q$ tandem mirror system using direct conversion. In such systems the end-loss fluxes would be larger and the plug pressures lower than those of a high- $Q$ system, thereby enhancing the effectiveness of the auto-stabilization mechanism.

The message from these various examples is that the combination of an axisymmetric tandem mirror with Ryutov's principle of MHD stabilization by the presence of plasma in an expander region is a very powerful one, opening up many new possibilities for tandem mirror fusion power systems.

\section{RESULTS FROM THE FLORA MHD-} STABILITY CODE
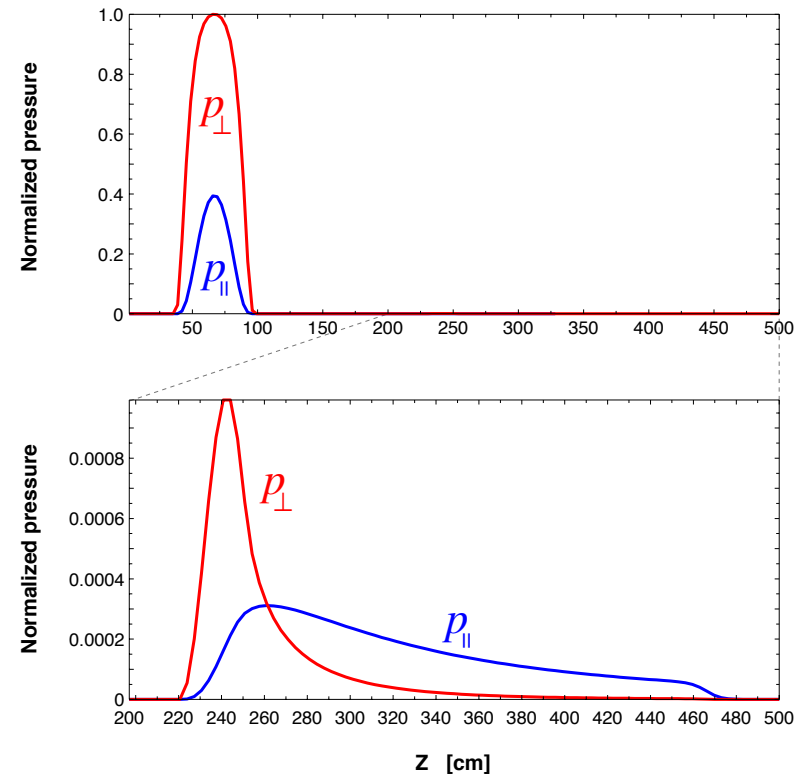

To benchmark our stability integral code at low beta and to allow the calculation of K-S T-M systems with high beta values in the plugs and central cell, we have updated and improved the FLORA MHD-stability code. This code, particularly in its time-dependent mode of operation, allows the analysis of the stability of axysymmetric equilibria, including finite-orbit effects, and "mirror," "firehose" and "ballooning" modes. An example of the benchmarking of FLORA against our low beta stability integral code is shown in Figure 8, which presents plots of $p_{\text {par }}$ and $p_{\text {perp }}$ for the plug plasma and the $\mathrm{K}-\mathrm{S}$ plasma for a marginally stable case. As can be seen there is close agreement between the codes, with the slight differences arising from the slightly different "normal mode" distributions in the plug that were used in the two codes.

Figure 8. Comparison plots of $p_{\text {perp }}$ and $p_{\text {par }}$ in the plug and the expander as evaluated for a marginally stable case by FLORA (left) and by the stability-integral code (right).

Using FLORA a search was instituted to find MHDstable high-beta cases based on the use of the K-S ion injection technique. It was found that at high beta the angular distribution of the injected K-S ions was an important parameter. Those cases where the beta value of the K-S plasma was elevated near the point of injection achieved the highest beta values, consistent with avoidance of the "firehose" mode near the point of injection (where that mode is most likely to arise). An example of such a case, with a plug beta stable at a value of $40 \%$, is shown in Figure 9. Although the beta values in the plug and the outer part of the expander are comparable, the fact that the magnetic field far out in the expander is much weaker (orders of magnitude) than that in the plug means that the K-S plasma pressure in this part of the expander is also many orders of magnitude smaller than that of the plug plasma. Also, since this FLORA calculation was performed using a simple expander field, i.e., the fringing field from the plug mirror field, it does not represent the optimized types of expander field that we have explored with our low-beta stability integral code. Using that code it was found possible to decrease the relative beta values (between the K-S plasma and the plug) by an order of magnitude while maintaining 
virtually the same stabilization ratio. In future studies FLORA will be reprogrammed to allow the investigation of more general types of expander fields.

The Kinetic Stabilizer ion injection parameters for the case shown in Figure 9 were made up of a skewed angular distribution (between the angles of 3 and 10 degrees relative to the field line direction at injection. To this distribution was then added a small (approximately 25 percent) constant wide-angle component, which results primarily in raising the $p_{\text {perp }}$ fraction at injection to be comparable to $p_{\text {par }} / 2$. This added $p_{\text {perp }}$ at injection both significantly reduces the net $B$ field at injection and increases the value of the field-line curvature, the combination of which results in a large contribution to the net stabilization of curvature-driven MHD interchange modes. In addition, the resulting sum of $p_{\text {perp }}$ and $p_{\text {par }}$ at injection is small enough to satisfy firehose stability. Figure 10 presents plots of $p_{\text {perp }}$ and $p_{\text {par }}$ within the plug (left plot) and the same pressure components of the K-S plasma (right plot). The plug plasma distribution is a "sloshing" ion distribution (suppresses the Alfven Ion Cyclotron (AIC) instability mode).

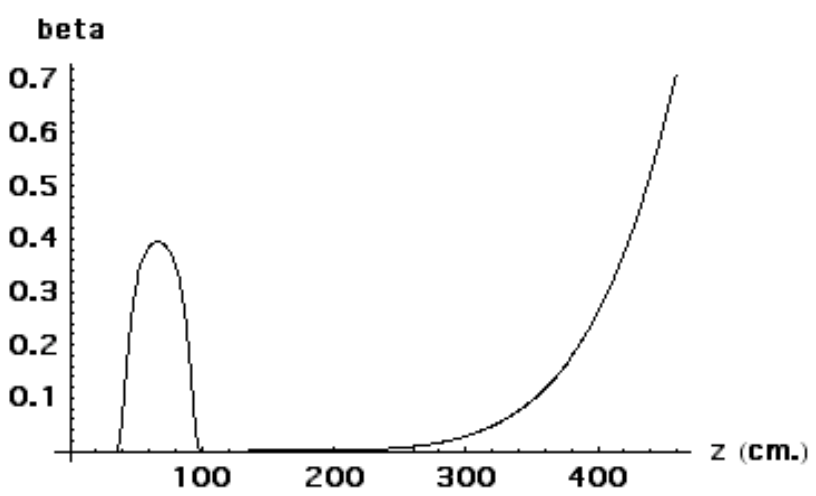

Figure 9. Plot of beta values in the plug and the expander for a case predicted by FLORA to be stable against all MHD modes at beta $=40$ percent in the plug
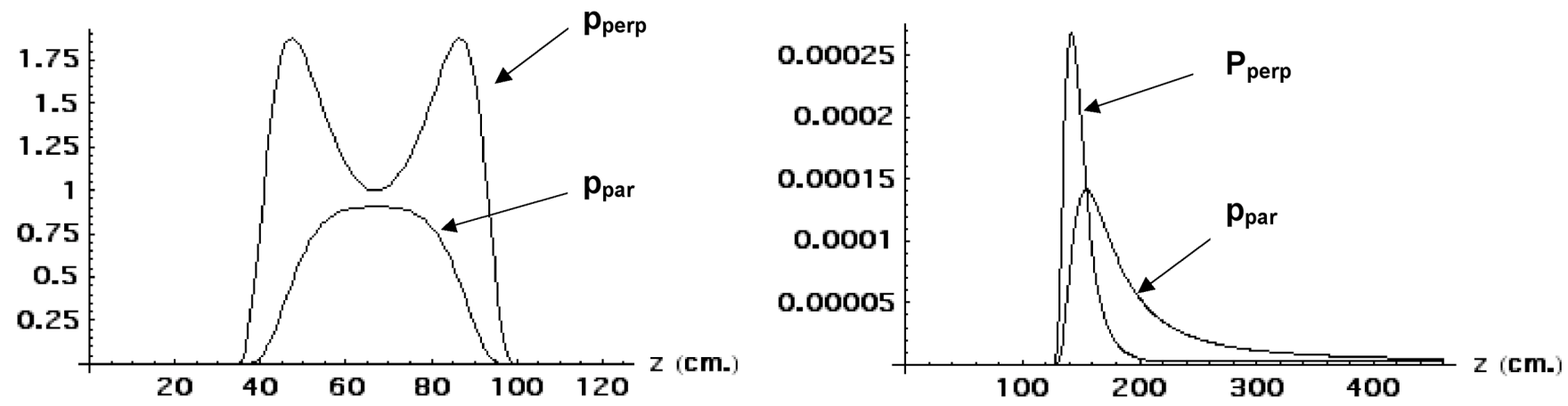

Figure 10 Plots of $p_{\text {perp }}$ and $p_{\text {par }}$ in the plug (left plot) and in the expander (right plot). The distribution in the plug is a "sloshing ion" distribution, with $p_{\text {perp }}$ normalized to unity at the midplane of the plug mirror field. Note that the peak K-S plasma pressure needed to stabilize the plug plasma is almost four orders of magnitudes smaller than the peak plug plasma

pressure.

\section{SUMMARY AND CONCLUSIONS}

This report has summarized the most recent results of an ongoing study of axisymmetric tandem mirror systems stabilized by the presence of plasma in the "expander" region outside the outermost plug mirror. The analyses have included the use of three computer codes, two of which were developed especially for these studies. One of these specially written codes, using the Mathematica ${ }^{\circledR}$ platform, was used to help optimize the design of the expander fields and to calculate MHD-stability integrals for the expander and for the plug and mirror cells. The second of these new codes, SYMTRAN, is a coupled radial-axial transport code that contains most of the important transport physics relevant to the K-S T-M, including a provision for ETG-induced radial transport. The third code employed, FLORA, is a MHD-stability code, updated and modified to investigate MHD modes in an axisymmetric tandem mirror system and their stabilization by the K-S plasma.

The findings to date concerning the K-S T-M are very encouraging as to its future. For example, SYMTRAN runs have found conditions for D-T ignition and stable burn with (un-optimized) fusion $Q$ values of 10 , achieved using a T-M central cell radius of 1.0 meter and a field of 3.0 Tesla. FLORA runs have confirmed the stabilization of plug plasmas with beta values of 40 percent, using K-S plasmas whose pressures are many orders of magnitude lower than those in the plug cell. The stability integral code has been used to explore a wide variety of expander field designs and different modes of generating the K-S plasma.

In the course of these studies we have also examined, preliminarily, the issue of residual instability modes and their suppression. It is believed that drift modes in the KS T-M can be stabilized by control of the radial potential 
distribution, as has already been shown in the Gamma 10 experiment at Tsukuba, Japan. The instability mode that we are now examining is the "trapped-particle" mode, one that might occur if the bridging plasma between the confined plasma and the K-S plasma is not sufficiently conductive. No evidence of this mode was seen in the GDT, but its stabilizing plasma varied monotonically in density between the confined plasma and the expander region. While the GDT example is encouraging to us that trapped-particle modes can be avoided in the K-S T-M by taking care in the formation of the K-S plasma, this issue is not yet resolved.

Taken overall, however, we feel that the K-S T-M has the potential to become a fusion power system with many desirable features. It is intrinsically a steady-state system and from an engineering standpoint it would appear to be much simpler and faster to implement than present mainline approaches. Its simplicity translates to the potential for its development and its deployment at a substantially lower capital cost than that of the mainline systems. Finally, in common with other open-ended systems, the K-S T-M has an inherent adaptability to innovative improvements, such as the introduction of direct conversion of escaping unburned fuel ions and charged reaction products. These many attributes make it a candidate for the fulfillment of the present most pressing need of the world magnetic fusion research effort, an early demonstration of the achievement of net power from a fusion device based on magnetic confinement.

\section{ACKNOWLEDGMENTS}

The authors would like to specially thank D. D. Ryutov for discussions with him of important plasmaphysics aspects of this paper. We also acknowledge continuing support from B. I. Cohen in helping with the FLORA code.

This work was performed under the auspices of the U. S. Department of Energy by the University of California National Laboratory under Contract W-7405ENG-48.

\section{REFERENCES}

[1] R. F. Post, Trans. of Fusion Technology, 39, 25 (2001)

[2] D. D. Ryutov, "Axisymmetric MHD-Stable Mirrors," Proceedings of the Course and Workshop, Varenna, Italy, Vol. II, 791 (1987)

[3] A. A. Ivanov, G. F. Abdrashitov, A. V. Anikiev, et. al., Trans. of Fusion Technology, 43, 51 (2003)

[4] G. I. Dimov, V. V. Zakaidakov, M. E. Kishinevskii, Sov. J. Plasma Physics, 2, 326 (1976)
[5] T. K. Fowler, B. G. Logan, Comments Plasma Phys. Controll. Fusioon, 2, 167 (1977)

[6] R. F. Post, Trans. of Fusion Technology, 35, 40 (1999)

[7] B. I. Cohen, R. P. Freis, W. A. Newcomb, Phys. Fluids, 29, 1558 (1986).

[8] R. H. Cohen, M. E. Rensink, T. A. Cutler, A. A. Mirin, Nucl. Fusion, 18, 1059 (1978)

[9] R. F. Post, Trans. of Fusion Technology, 43, 195 (2003)

[10] R. F. Post, Plasma Physics Reports, 28, 712 (2002)

[11] R. H. Cohen, Nuclear Fusion, 19, 1295 (1979)

[12] F. H. Coensgen, et. al., Phys. Rev. Letters, 44, 1132 (1980)

[13] T. C. Simonen, et. al., "Plasma Confinement Experiments in the TMX Tandem Mirror," Brussels IAEA, 1980, paper IAEA-CN-38/F-1

[14] A. A. Mirin, S. P Auerbach, R. H. Cohen, et. al., Nuclear Fusion, 23, 703 (1983)

[15] V. V. Mirnov, D. D. Ryutov, "Gas Dynamic Trap," in Plasma Physics, Vol. 8 (Summaries I Science and Technology, V.D. Shafranov, Ed.), State Institute for Scientific Information, Moscow, 1988 (in Russian)

[16] D. D. Ryutov (private communication)

[17] R. F. Post, D. D. Ryutov, in Current Trends in International Fusion Research, Ed. E. Panarella, 153, Plenum Press, New York (1997) 